\title{
Variantes Alpha y Gamma del SARS-CoV-2: revisión rápida para contribuir en la toma de decisiones
}

\section{Alpha and Gamma variants of SARS-CoV-2: rapid review to aid decision-making}

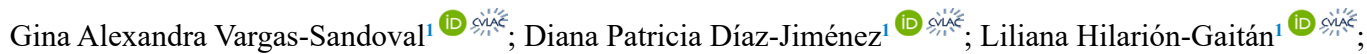

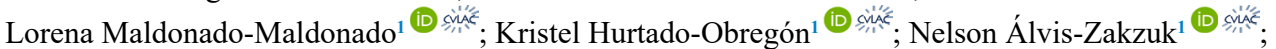

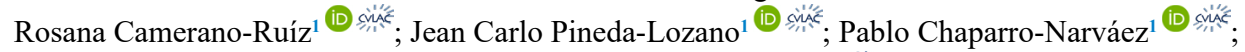

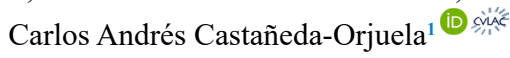

Forma de citar: Vargas Sandoval GA, Díaz Jiménez D, Hilarión Gaitán L, Maldonado Maldonado L, Hurtado Obregón K, Álvis Zakzuk N, et al. Variantes Alpha y Gamma del SARS-CoV-2: revisión rápida para contribuir en la toma de decisiones. Salud UIS. 53: e21023. doi: https://doi.org/10.18273/saluduis.53.e:21023 (c) (i)

\section{Resumen}

Objetivo: describir el riesgo de transmisión y severidad de las variantes de preocupación Alpha y Gamma del SARS-CoV-2 en comparación con otras variantes. Materiales y métodos: revisión rápida y narrativa de literatura realizada en PubMed hasta mayo 10 de 2021. Resultados: la búsqueda capturó 262 artículos, de los cuales se incluyeron 15 estudios. Cuatro fueron incluidos en el control de referencias. De los 19 estudios, 6 corresponden con literatura gris. La mayoría de los estudios analizaron la variante de preocupación Alpha, solo uno para Gamma. La variante de preocupación Alpha es consistente con mayor riesgo de transmisión (entre el 35 y 89\% mayor riesgo), de hospitalización (entre 64 y $234 \%$ mayor riesgo) y de muerte (entre 36 y $140 \%$ mayor riesgo) en comparación con otras variantes. La variante de preocupación Gamma reporta una posible correlación fuerte y positiva entre proporción positivos y las muertes (Rho 0,$71 ; \mathrm{p}<0,01)$. Conclusiones: se encuentra más información de la variante de preocupación Alpha que de Gamma. La variante de preocupación Alpha reporta mayor riesgo de transmisión, hospitalización y muerte en comparación con otras variantes; pero estos resultados deben tomarse con precaución.

Palabras clave: Coronavirus; Infecciones por Coronavirus; Transmisión; Hospitalización; Cuidados Críticos; Mortalidad.

\begin{abstract}
Objective: To describe the transmission and severity risk of the Alpha and Gamma variants concerning SARSCoV-2 compared with other variants. Materials and methods: Narrative and rapid review conducted in PubMed up to May 10, 2021. Results: The search captured 262 articles, of which 15 studies were included. Four studies were

\footnotetext{
1. Instituto Nacional de Salud. Bogotá, Colombia

Correspondencia: Gina Alexandra Vargas Sandoval. Dirección: Instituto Nacional de Salud; Av. calle 26 51-20 - Zona 6 CAN. Bogotá, D.C. Teléfono: +57 (1) 220 7700. Correo electrónico: gvargas@ins.gov.co
} 
included from the reference control. Six out of the 19 studies were gray literature. Most of the studies analyzed the variant of concern, Alpha, and only one for the Gamma variant. The variant of concern, Alpha, is consistent with a higher risk of transmission (between 35 and 89\% higher risk), hospitalization (between 64 and 234\% higher risk) and death (between 36 and 140\% higher risk) compared to other variants. The Gamma variant reports a possible strong and positive correlation between positive proportions and deaths (Rho 0.71; $<<0.01$ ). Conclusions: There is more information regarding the variant of concern Alpha than Gamma. The variant of concern Alpha reports a higher risk of transmission, hospitalization, and death; but these results should be viewed with caution.

Keywords: Coronavirus; Coronavirus Infections; Transmission; Hospitalization; Critical Care; Mortality.

\section{Introducción}

A mayo de 2021 en el mundo se han registrado más de 170 millones de casos confirmados y 3,5 millones de muertes por SARS-Cov- $2^{1}$. La crisis sanitaria, social y económica derivada de la pandemia podría agudizarse entre otras cosas, por los mecanismos adaptivos del SARS-CoV2, como las mutaciones emergentes que podrían significar mayor transmisibilidad y severidad en los seres humanos ${ }^{2}$.

La Organización Mundial de la Salud (OMS) ha identificado tres variantes de preocupación (VOC, por sus siglas en inglés) del SARS-CoV-2, detectadas por primera vez en Reino Unido (Alpha, 20I/501Y.V1, VOC 202012/01, o B.1.1.7), Sudáfrica (Beta, B.1.351/501. YV2) y Japón en viajeros provenientes de Brasil (Gamma, B.1.1.248/B1.1.28/P1) ${ }^{3}$. Las VOC se definen como variantes que reportan mayor transmisibilidad, severidad o demandan cambios en las intervenciones para su control ${ }^{4}$.

En Colombia, se identificó que la VOC Alpha circula desde el 15 de febrero de 2021 y VOC Gamma desde el 4 de enero de 2021. De los 1857 genomas secuenciados el $18,0 \%$ y $4,57 \%$ corresponden con VOC Alpha y Gamma, respectivamente ${ }^{5}$. Por lo anterior se hace urgente proporcionar información que permita tomar decisiones en salud pública correspondientes con este nuevo contexto. Se realizó una revisión de literatura narrativa y rápida con el objetivo de describir el riesgo de transmisión y severidad de las VOC Alpha y Gamma del SARS-CoV-2 en comparación con otras variantes.

\section{Materiales y métodos}

Se realizó una revisión rápida y narrativa de literatura, en el motor de búsqueda de PubMed, con la sintaxis de búsqueda (variant*[title/abstract] AND ("20I/501Y. V1"[title/abstract] OR “202012/01”[title/abstract] OR “B.1.1.7”[title/abstract] OR "P.1"[title/abstract] OR UK[title/abstract] OR "united kingdom"[title/ abstract] OR brazil*[title/abstract])) AND ((“covid 19"[MeSH Terms] OR coronavirus[title/ abstract] OR coronavirus'[title/abstract] OR coronavirus's[title/abstract] OR coronavirus[title/ abstract] OR coronavirus*[title/abstract] OR coronaviruses[title/abstract] OR coronaviruses'[title/ abstract] OR "coronavirus like"[title/abstract]) OR ((covid[title/abstract] OR corvid[title/abstract]) AND 19[title/abstract]) OR (novel[title/abstract] AND coronavirus[title/abstract]) OR (covid[title/abstract] AND2[title/abstract]) OR “COVID-19"[Supplementary Concept] OR "severe acute respiratory syndrome coronavirus 2"[Supplementary Concept]).

Se incluyeron estudios originales, literatura indexada o gris (preprint y reportes de entidades gubernamentales), que estimaran el riesgo transmisión o severidad de las VOC Alpha y Gamma en comparación con otras variantes. Se excluyeron reportes de caso y parámetros estimados en modelos predictivos; estudios de reinfecciones, validación de pruebas diagnósticas y estudios en animales. Se realizó control de referencias de los estudios analizados, con el objetivo de obtener estudios adicionales no capturados por la estrategia de búsqueda.

En una plantilla de Microsoft ${ }^{\circledR}$ Excel previamente estandarizada, se describieron datos generales de los estudios como diseño(estudios ecológicos, transversales, de cohorte, series de casos, casos y controles), características de la población de estudio como país, grupos de edad, número y tipo de casos (comunitarios u hospitalizados); métodos para determinar la circulación o infección por VOC y grupos de comparación. Se extrajeron parámetros epidemiológicos de riesgo de transmisión y severidad VOC en comparación con otras variantes; y letalidad por VOC. La revisión de título y resumen; y extracción de datos se hizo por pares independientes. Las discrepancias fueron resueltas por un tercer revisor. Por tratarse de una revisión narrativa y rápida, no se realizó evaluación de calidad de los estudios. 
La síntesis de los estudios se realizó con los parámetros hallados por cada VOC. Se muestran los valores de los parámetros de frecuencia y asociación, describiendo la heterogeneidad entre población de estudio y metodológica entre los estudios que se consideraron de mayor relevancia a criterio de los revisores. Esta revisión no requirió aprobación del comité de ética por ser análisis de información secundaria ya publicada sin identificación personal.

\section{Resultados}

La sintaxis de búsqueda capturó 262 artículos, de los cuales se preseleccionaron 51 para lectura de texto completo. Una vez verificados los criterios de selección, fueron incluidos 19 estudios, cuatro capturados en el control de referencias (Figura 1). Las características generales de los estudios incluidos en este análisis se muestran en la Tabla 1. Al momento del análisis de esta revisión, dos estudios permanecían en preprint $t^{6,7}$ y cuatro $^{8-11}$ como reportes no publicados. Todos los artículos analizaron la VOC Alpha ${ }^{6,7,16-23,8-15}$, excepto uno de la VOC Gamma ${ }^{24}$. Se reportaron estimadores de transmisión ${ }^{12,14,19}$, severidad ${ }^{6,7,12-17,22,23}$ y muerte ${ }^{8-11,18,20-24}$. El porcentaje de participantes confirmados con VOC se registró entre 11,6 y $75 \%$.

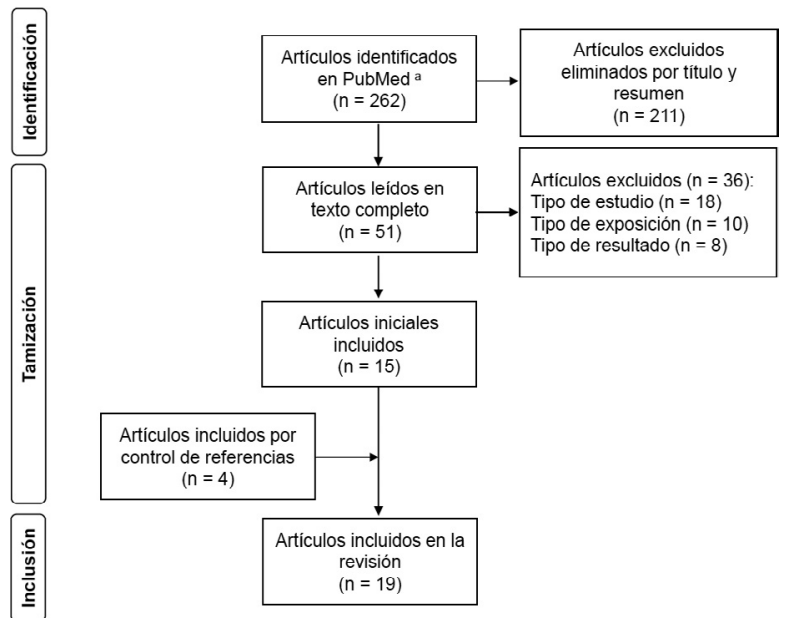

Figura 1. Diagrama de flujo de la selección de artículos.

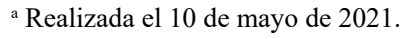

Tabla 1. Características generales de los estudios incluidos en el análisis según VOC.

\begin{tabular}{|c|c|c|c|c|c|c|c|}
\hline \multirow{2}{*}{ Estudio } & \multirow{2}{*}{ País } & \multirow{2}{*}{ Diseño } & \multirow{2}{*}{ Participantes } & \multirow{2}{*}{$\begin{array}{c}\text { Porcentaje de } \\
\text { participantes con VOC }\end{array}$} & \multicolumn{3}{|c|}{ Parámetros } \\
\hline & & & & & Trans. $^{\mathrm{a}}$ & Sev. $^{\mathrm{b}}$ & Let. \\
\hline \multicolumn{8}{|l|}{ Variante inglesa } \\
\hline Graham, $2021^{6}$ & Reino Unido & Ecológico & $\begin{array}{c}\text { Trans.: } 98170 \\
\text { Sev.: } 36920\end{array}$ & $\begin{array}{c}\text { Trans.: } 16,5 \% \\
\text { Sev.: } 34,6 \%\end{array}$ & $\mathrm{X}$ & $\mathrm{X}$ & $N^{f}$ \\
\hline Brown, $2021^{7}$ & Toronto, Canadá & Ecológico & 20051 & $23,40 \%$ & $X$ & NR & NR \\
\hline Volz, $2021^{8}$ & Inglaterra & Ecológico & 84185 & $37,20 \%$ & $\mathrm{X}$ & NR & NR \\
\hline Grabowski, $2021^{9}$ & $\begin{array}{l}\text { Reino Unido, USA, } \\
\text { Dinamarca }\end{array}$ & Ecológico & NR & NR & $\mathrm{X}$ & NR & NR \\
\hline Davies, $2021^{10}$ & $\begin{array}{l}\text { Reino Unido, Dinamarca, } \\
\text { Suiza y USA }\end{array}$ & Ecológico & NR & NR & $\mathrm{X}$ & NR & NR \\
\hline Garvey, $2021^{11}$ & Inglaterra & Series de casos & 152 & $51,90 \%$ & NR & $\mathrm{X}$ & $\mathrm{X}$ \\
\hline Loconsole, $2021^{12}$ & Apulia, Italia & Transversal & 3075 & $20,20 \%$ & NR & $\mathrm{X}$ & $\mathrm{X}$ \\
\hline Bager, $2021^{13 d}$ & Dinamarca & Cohorte & 18499 & $11,60 \%$ & NR & $\mathrm{X}$ & NR \\
\hline Brookman, $2021^{14 d}$ & Londres, Inglaterra & Serie de casos & 80 & $75,00 \%$ & NR & $\mathrm{X}$ & NR \\
\hline Grint, $2021^{15}$ & Inglaterra & Cohorte & 184786 & $50,30 \%$ & NR & $\mathrm{X}$ & NR \\
\hline Frampton, $2021^{16}$ & Londres, Inglaterra & Cohorte & 341 & $58,00 \%$ & NR & $\mathrm{X}$ & NR \\
\hline Davies, $2021^{17}$ & Inglaterra & Transversal & 1146534 & $58,8 \%$ & NR & NR & $\mathrm{X}$ \\
\hline Docherty, $2021^{18 \mathrm{e}}$ & Reino Unido & $\begin{array}{l}\text { Reporte de casos } \\
\text { Ecológico }\end{array}$ & $\begin{array}{c}214 \\
108116\end{array}$ & $14,9 \% \mathrm{NR}$ & NR & NR & $\mathrm{X}$ \\
\hline Calistri, $2021^{19}$ & Abruzzo, Italia & Transversal & 2657 & $11,70 \%$ & NR & NR & $\mathrm{X}$ \\
\hline Ferguson, $2021^{20 \mathrm{e}}$ & Inglaterra & $\mathrm{SD}$ & 120000 & NR & NR & NR & $\mathrm{X}$ \\
\hline Challen, $2021^{21}$ & Reino Unido & Cohorte pareada & 109812 & $50 \%$ & NR & NR & $\mathrm{X}$ \\
\hline PHE, $2021^{22 \mathrm{e}}$ & Inglaterra & Cohorte & NR & NR & NR & NR & $\mathrm{X}$ \\
\hline $\begin{array}{l}\text { Universidad Exeter, } \\
2021^{23 \mathrm{e}}\end{array}$ & Inglaterra & $\begin{array}{l}\text { Casos y controles } \\
\text { pareado }\end{array}$ & NR & NR & NR & NR & $\mathrm{X}$ \\
\hline \multicolumn{8}{|l|}{ Variante brasileña } \\
\hline Zimerman, $2021^{24}$ & Amazonas, Brasil & Ecológico & 77 & NR & NR & NR & $\mathrm{X}$ \\
\hline
\end{tabular}

${ }^{\mathrm{a}}$ Transmision, ${ }^{\mathrm{b}}$ Severidad, ${ }^{\mathrm{c}}$ Letalidad, ${ }^{\mathrm{d}}$ preprint, ${ }^{\mathrm{e}}$ Datos no publicados, ${ }^{\mathrm{f}}$ No reportado. 
La mayoría de estudios fueron ecológicos ${ }^{12-15,19,24}$, seguidos por análisis de cohortes ${ }^{6,10,16,17,21}$, estudios transversales ${ }^{18,20}$, series de $\operatorname{casos}^{7,8}$ y uno de casos y controles ${ }^{11}$. Los estudios se realizaron con casos identificados por la tamización en la población general (casos comunitarios) $)^{6,8,18-22,24,9-16} \mathrm{u}$ hospitalizados y confirmados para COVID-19 (hospitalarios) ) $^{7,8,17,23}$. Los métodos diagnósticos para la determinación de la variante (VOC u otras variantes) fueron la secuenciación genómica $(\mathrm{SG})^{12-14,19}$, SG completa ${ }^{6,17,23,24}$, marcador SGTF (por sus siglas en inglés, falla en la detección de la mutación del gen de la espiga) 9,10,20-22,11-16,18,19 o por picos epidemiológicos ${ }^{7,8}$. Algunos estudios usaron más de un método.

\section{VOC Alpha}

Se hallaron parámetros para transmisión ${ }^{12-15,19}$, severidad $^{6,7,12,17,22,23}$ y letalidad ${ }^{8-11,18,20-23}$.

\section{Transmisión}

Cinco estudios ecológicos sobre casos comunitarios reportaron la ventaja de transmisión de VOC Alpha (Tabla 2), realizados en Reino Unido ${ }^{12,14,15,19}$, USA ${ }^{15,19}$, Canadá $^{13}$, Dinamarca ${ }^{19}$ y Suiza ${ }^{19}$; entre octubre de 2020 y marzo de 2021, fechas con crecimiento del contagio por VOC Alpha en dichos países. Para la identificación de las variantes se usaron la $\mathrm{SG}^{12,14,15,19}$ o por marcador SGTF ${ }^{12-14,19}$. En consecuencia, los grupos de comparación se configuraron a partir de variantes que no correspondieron con el genoma VOC Alpha ${ }^{12,14,15,19}$ y variantes $\sin$ marcador SGTF $^{12-14,19}$. La ventaja en la transmisión por VOC Alpha se estimó entre 25-89\% en comparación con otras variantes. En consideración a los modelos implementados, se reportaron para las estimaciones puntuales intervalos de confianza (mixtos lineales generalizados) (IC95\%) o de credibilidad (dinámicos de transmisión) (ICr 95\%), siendo estos superiores al valor nulo (rango entre 1,02 - 2,65\%).

Tabla 2. Ventaja en la transmisión para VOC-I ${ }^{\mathrm{a}}$ en comparación con otras variantes reportados en estudios revisados, según método de determinación de variantes.

\begin{tabular}{|c|c|c|c|c|}
\hline País & Periodo & $\begin{array}{l}\text { Ventaja en la transmisión } \\
\text { Valor }\left(\operatorname{IC}^{\mathrm{b}} \text { o ICr } \operatorname{ICr}^{\mathrm{c}} \mathbf{9 5}\right)\end{array}$ & Variantes de referencia & Ref. \\
\hline \multicolumn{5}{|l|}{ Secuenciación genómica } \\
\hline Reino Unido & Feb./20 a Ene. $/ 21$ & $1,77(1,73-1,81)$ & 20A.EU1 & 10 \\
\hline Escocia, Gales e Inglaterra & Oct./20 a Nov./20 & $1,35(1,02-1,69)$ & No VOC-I & 6 \\
\hline Escocia, Gales e Inglaterra & Dic. $/ 20$ & $1,28(1,02-1,61)$ & No VOC-I & 6 \\
\hline Inglaterra & Oct./ 20 a Ene./21 & $1,89(1,43-2,65)^{\mathrm{c}}$ & No VOC-I & 8 \\
\hline Inglaterra & Ene./21 & $1,54(1,34-1,82)^{\mathrm{c}}$ & No VOC-I & 8 \\
\hline Inglaterra & Oct./20 a Nov./20 & $2,47(1,89-2,77)$ & No VOC-I ni 20A.EU1 & 9 \\
\hline Inglaterra & Oct./20 a Nov./20 & $2,18(1,97-2,40)$ & 20A.EU1 & 9 \\
\hline Inglaterra & Oct./20 a Dic./20 & $1,83(1,71-1,96)$ & 20A.EU1 & 9 \\
\hline Gales & Nov./20 a Ene./21 & $1,65(1,46-2,04)$ & No VOC-I & 9 \\
\hline Dinamarca & Oct./20 a Ene./21 & $1,55(1,45-1,66)$ & No VOC-I & 10 \\
\hline Suiza & Nov./20 a Feb./21 & $1,74(1,66-1,82)$ & No VOC-I & 10 \\
\hline USA & Nov./20 a Ene./21 & $1,72(1,46-2,04)$ & No VOC-I & 9 \\
\hline \multicolumn{5}{|l|}{ Marcador SGTF ${ }^{\mathrm{d}}$} \\
\hline Inglaterra & Sep./20 a Dic./20 & $1,25(1,17-1,34)$ & No SGTF & 6 \\
\hline Inglaterra & Oct./20 a Ene./21 & $1,82(1,43-2,30)^{\mathrm{c}}$ & No SGTF & 10 \\
\hline Inglaterra & Oct./20 a Ene./21 & $1,83(1,81-1,84)$ & No SGTF & 10 \\
\hline Inglaterra & Nov./20 a Ene./21 & $1,79(1,22-2,49)$ & No SGTF & 8 \\
\hline Inglaterra & Nov./20 a Ene./21 & $1,60(1,09-2,23)$ & No SGTF & 8 \\
\hline Toronto, Canadá & Dic./20 a Mar./21 & $1,34(1,31-1,38)$ & No SGTF & 7 \\
\hline USA & Sep./20 a Feb./21 & $1,59(1,56-1,83)$ & No SGTF & 10 \\
\hline
\end{tabular}

${ }^{\mathrm{a}}$ Variante de preocupación inglesa, ${ }^{\mathrm{b}}$ Intervalos de confianza, ${ }^{\mathrm{c}}$ Intervalos de credibilidad, ${ }^{\mathrm{d}}$ Falla en la detección de la mutación del gen de la espiga. 
La ventaja en la transmisión de VOC Alpha se mantuvo incluso con algunas diferencias en la población y métodos entre los estudios. Por ejemplo, a pesar de que la mayoría de estimaciones se hicieron para Reino Unido e Inglaterra ${ }^{12,14,15,19}$, la transmisión fue también mayor en los demás países analizados ${ }^{13,15,19}$. Algunos estudios reportaron disminución de la ventaja en la transmisión de VOC Alpha al final de la serie analizada, en algunos casos para el periodo posterior al confinamiento (pasando del $35 \%$ al 28\%) ${ }^{12}$; o relacionándose con el incremento de la proporción de contagios por VOC Alpha (pasando del $89 \%$ al $54 \%)^{14}$; y del $118 \%$ al $83 \%{ }^{15}$. La ventaja en la transmisión se mantuvo al comparar contra variantes específicas como con 20A. EU1 ${ }^{15,19}$ o no VOC Alpha ni 20A.EU1 ${ }^{15}$, tendiendo a reportar ventajas mayores (superiores al $77 \%$ ).

\section{Severidad}

Seis estudios analizaron la severidad de la enfermedad por VOC Alpha ${ }^{6,7,12,17,22,23}$ (Tabla 3). Tres estudios en casos comunitarios estimando el riesgo de hospitalización ${ }^{6,12,22}$; y el resto sobre casos hospitalarios $^{7,17,23}$. Todos los estudios se hicieron en población general, excepto uno en menores de edad'. La determinación de la VOC Alpha se hizo por SG completa ${ }^{6,17,23}$, marcador $\mathrm{SGTF}^{22}$, SG completa y marcador SGTF combinado ${ }^{12}$ y por comparación entre el segundo y primer pico epidemiológico de COVID-19 asumiendo mayor circulación de la VOC Alpha en el segundo pico $^{7}$.

Tabla 3. Estimadores de asociación para severidad entre VOC-I en comparación con otras variantes según tipos de caso.

\begin{tabular}{|c|c|c|c|c|c|c|c|}
\hline \multirow[b]{2}{*}{ Desenlace } & \multirow{2}{*}{$\begin{array}{l}\text { Determinación } \\
\text { de variantes }\end{array}$} & \multicolumn{3}{|c|}{ Estimadores de frecuencia } & \multirow{2}{*}{\multicolumn{2}{|c|}{$\begin{array}{l}\text { Estimadores de } \\
\text { asociación valor } \\
\text { (IC } 95 \% \text { ) }\end{array}$}} & \multirow[b]{2}{*}{ Ref. } \\
\hline & & Estimador & VOC-I & Otras variantes & & & \\
\hline \multicolumn{8}{|l|}{ Casos comunitarios } \\
\hline Hospitalización & $\mathrm{SGTF}^{\mathrm{b}}$ & & $\mathrm{NR}^{\mathrm{d}}$ & & OR & $3,34(1,76-6,75)$ & 13 \\
\hline Hospitalización & $\mathrm{SGC}^{\mathrm{c}}$ & & $\mathrm{NR}$ & & OR & $1,64(1,32-2,91)$ & 13 \\
\hline 0-29 años & $\mathrm{SGC}$ & & NR & & OR & $1,84(1,01-3,35)$ & 13 \\
\hline 30-59 años & $\mathrm{x}$ & & NR & & OR & $1,62(1,18-2,23)$ & 13 \\
\hline$>60$ años & SGC & & NR & & OR & $1,61(1,16-2,23)$ & 13 \\
\hline Infección sintomática & SGTF & Porcentaje & $51,7 \%$ & $39,3 \%$ & & $\mathrm{NR}$ & 12 \\
\hline \multicolumn{8}{|l|}{ Casos hospitalizados } \\
\hline Enfermedad grave o muerte & SGC & & NR & & $\mathrm{RP}$ & $1,02(0,76-0,38)$ & 16 \\
\hline Enfermedad grave & SGC & & NR & & RP & $1,30(0,97-1,74)$ & 16 \\
\hline Días estancia hospitalaria & SGC & Promedio & 9,5 & 11,5 & & $\mathrm{NR}$ & 11 \\
\hline Días UCI & SGC & Promedio & 18,9 & 15,2 & & NR & 11 \\
\hline Enfermedad crítica & $2^{\text {do }}$ pico & Porcentaje & $3,0 \%$ & $20,0 \%$ & & NR & 14 \\
\hline Admisión UCI & SGC & Porcentaje & $19,0 \%$ & $11,1 \%$ & & NR & 11 \\
\hline Ventilación invasiva & $2^{\text {do }}$ pico & Porcentaje & $2,0 \%$ & $20,0 \%$ & & NR & 14 \\
\hline Ventilación no invasiva & $2^{\text {do }}$ pico & Porcentaje & $3,0 \%$ & $15,0 \%$ & & NR & 14 \\
\hline Enfermedad moderada & $2^{\text {do }}$ pico & Porcentaje & $13,0 \%$ & $25,0 \%$ & & NR & 14 \\
\hline Enfermedad severa & $2^{\text {do }}$ pico & Porcentaje & $5,0 \%$ & $5,0 \%$ & & NR & 14 \\
\hline Enfermedad leve & $2^{\text {do }}$ pico & Porcentaje & $45,0 \%$ & $40,0 \%$ & & NR & 14 \\
\hline Enfermedad asintomática & $2^{\text {do }}$ pico & Porcentaje & $33,0 \%$ & $10,0 \%$ & & NR & 14 \\
\hline Tos & SGC & Porcentaje & $59,0 \%$ & $38,0 \%$ & & NR & 11 \\
\hline
\end{tabular}

${ }^{a}$ Variante de preocupación inglesa, ${ }^{b}$ Falla en la detección de la mutación del gen de la espiga, ${ }^{\mathrm{c}}$ Secuenciación genómica completa, ${ }^{\mathrm{d}}$ No reportado, Diferenicas estadisticamente significativas $(\mathrm{p}<0.05)$.

Los estudios reportaron estimadores de frecuencia y de asociación para determinar la evidencia de severidad de la infección por VOC Alpha. Se encontraron diferencias estadísticamente significativas en contagiados con VOC Alpha, reportando mayor riesgo de hospitalización en

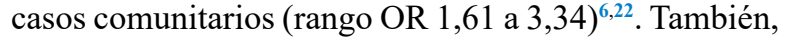
diferencias significativas entre porcentajes de infección sintomática y tos en hospitalizados ${ }^{22,23}$. Los demás desenlaces analizados no reportaron riesgo o diferencias significativas. Se reportó que el análisis de regresión 
lineal no mostró asociación entre la proporción de infección por la VOC Alpha y el autorreporte de síntomas, hospitalización o duración de síntomas mayor a 28 días, pero los valores de la estimación no fueron presentados $^{12}$.

\section{Muerte por VOC}

Quince estudios reportaron letalidad o riesgo de muerte por VOC Alpha (Tabla 4). Dos fueron en casos hospitalizados $^{8,23}$ y el resto en comunitarios ${ }^{8,9,11,16,18,20-22,25}$.
La determinación de la VOC Alpha se hizo en su mayoría por marcador $\mathrm{SGTF}^{9,11,16,18,20-22,25}, \mathrm{SGC}^{23}$, picos epidemiológicos ${ }^{8} \mathrm{y}$ en un caso no fue reportado ${ }^{8}$. Se realizó seguimiento hasta la muerte o 28 días desde el diagnóstico, excepto en dos estudios que no lo reportaron ${ }^{916}$. En población general, la letalidad por VOC Alpha se reportó entre $15-18 \%$ en casos hospitalarios $^{8,23}$ y 0,3-2,1\% entre comunitarios ${ }^{16,18,20-22,26}$. Los hombres de más edad reportaron la letalidad más alta ${ }^{18}$.

Tabla 4. Letalidad y riesgo de muerte para VOC-I $I^{a}$ en comparación con otras variantes según tipo de casos.

\begin{tabular}{|c|c|c|c|}
\hline Población & Letalidad Valor (IC $95 \%$ ) & Riesgo de muerte Estimador valor (IC $95 \%$ ) & Ref. \\
\hline \multicolumn{4}{|l|}{ Hospitalarios } \\
\hline General & $15,6 \%\left(\mathrm{NR}^{\mathrm{b}}\right)$ & OR $0,63(0,20-1,69)$ & 18 \\
\hline General & $18,0 \%(\mathrm{NR})$ & $\mathrm{NR}$ & 11 \\
\hline \multicolumn{4}{|l|}{ Comunitarios } \\
\hline General & $2,10 \%(0,8-6,1 \%)$ & NR & 19 \\
\hline General & $0,6 \%(\mathrm{NR})$ & NR & 12 \\
\hline General & $0,5 \%(\mathrm{NR})$ & NR & 15 \\
\hline \multirow{2}{*}{ General } & \multirow{2}{*}{$0,3 \%(\mathrm{NR})$} & HR $1,64(1,32-2,04)^{\mathrm{c}}$ & 21 \\
\hline & & HR $2,40(1,66-3,74)^{d}$ & 21 \\
\hline General & NR & HR $0,57(0,55-0,59)$ & 18 \\
\hline General & NR & RR $1,36(1,18-1,56)$ & 20 \\
\hline General & NR & HR $1,55(1,39-1,72)$ & 17 \\
\hline General & NR & RR $1,65(1,21-2,25)$ & 22 \\
\hline General & NR & HR $1,67(1,34-2,09)$ & 15 \\
\hline General & NR & RR 1,91 (1,35 - 2,71) & 23 \\
\hline \multicolumn{4}{|l|}{ Ambos sexos } \\
\hline $0 \mathrm{a}<65$ años & NR & HR 1,61 $(1,11-2,34)$ & 15 \\
\hline $65 \mathrm{a}<75$ años & NR & HR $1,64(1,15-2,36)$ & 15 \\
\hline $75 \mathrm{a}<85$ años & NR & HR $1,61(1,13-2,29)$ & 15 \\
\hline$\geq 85$ años & NR & HR $1,94(1,34-2,81)$ & 15 \\
\hline \multicolumn{4}{|l|}{ Mujeres } \\
\hline ene-34 & $0,0011 \%$ & NR & 17 \\
\hline $35-54$ & $0,05 \%$ & NR & 17 \\
\hline $55-69$ & $0,28 \%$ & NR & 17 \\
\hline $70-84$ & $4,4 \%$ & NR & 17 \\
\hline$>85$ & $19,0 \%$ & NR & 17 \\
\hline \multicolumn{4}{|l|}{ Hombres } \\
\hline ene-34 & $0,0047 \%$ & NR & 17 \\
\hline $35-54$ & $0,099 \%$ & NR & 17 \\
\hline $55-69$ & $0,86 \%$ & NR & 17 \\
\hline $70-84$ & $7,2 \%$ & NR & 17 \\
\hline$>85$ & $25,0 \%$ & NR & 17 \\
\hline
\end{tabular}

${ }^{\mathrm{a}}$ Variante de preocupación inglesa, ${ }^{\mathrm{b}}$ No reportado, ${ }^{\mathrm{c}} 1$ a 14 días, ${ }^{\mathrm{d}} 15$ a 28 días. Diferenicas estadisticamente significativas $(\mathrm{p}<0.05)$. 
Los casos hospitalizados no reportaron la infección por VOC Alpha como riesgo para la muerte ${ }^{8}$. Para casos comunitarios, todos los estudios informaron riesgos de muerte en infectados por VOC Alpha estadísticamente significativos ${ }^{9}, 11,16,18,20-22,25$, excepto un estudio ${ }^{8}$. El riesgo de muerte aumenta con la edad ${ }^{16}$ y entre $\operatorname{los} 15$ a 28 días de seguimiento ${ }^{21}$. Otros estudios no mostraron medidas de asociación, pero evidenciaron que no existían diferencias estadísticamente significativas entre la letalidad por VOC Alpha, en comparación con no VOC Alpha en casos hospitalarios ${ }^{23}$ y $\operatorname{comunitarios}^{22}$.

\section{VOC Gamma}

Solo un estudio ecológico con análisis de la VOC Gamma, desarrollado en la Amazonía brasileña ${ }^{24}$. Este analizó la correlación entre proporción positivos y muertes semanales por la VOC Gamma en ochos semanas, reportando un Rho de 0,71 (p <0,01) indicando una posible correlación fuerte y positiva entre estos dos indicadores. Para el final del periodo de análisis, Amazonas registraba el $87,5 \%$ de las muestras analizadas con la VOC Gamma. No se hallaron otros aspectos como transmisión o severidad para esta variante.

\section{Discusión}

Según esta revisión narrativa rápida de literatura, hay mayor frecuencia de estudios de la variante de preocupación Alpha (VOC Alpha) que de la Gamma (VOC Gamma). Los estudios relacionados con la VOC Alpha son consistentes en reportar mayor riesgo de transmisibilidad (entre el 35 y $89 \%$ mayor riesgo) $)^{12-15,19} \mathrm{y}$, en casos comunitarios, mayor riesgo de hospitalización (entre 64 y $234 \%$ mayor riesgo) $)^{6,22}$ y muerte (entre 36 y $140 \%$ mayor riesgo) ${ }^{9,11,16,18,21,25}$ en comparación con otras variantes.

Al respecto, un método usado para determinar la VOC Alpha fue el marcador SGTF el cual se ha considerado sensible para esta variante ${ }^{14}$, pero no necesariamente específico. De manera concreta, las estimaciones de asociación con desenlaces asociados a las VOC pueden ser espurias en consideración a que sea la mayor transmisión ocasionada por mayor interacción en regiones en las cuales la VOC Alpha es más frecuente y no necesariamente una ventaja en la transmisibilidad de la variante ${ }^{19}$. En adición, los datos usados en los estudios parten de la vigilancia rutinaria y no son representativos de los casos positivos de SARS-CoV-2 ${ }^{4}$.
En cuanto a la VOC Gamma, esta revisión mostró que no existen suficientes estudios publicados que permitan concluir sobre el riesgo de letalidad diferencial. Es probable que más estudios de la VOC Alpha se deban a que la mayoría de información proviene de países desarrollados y por tanto con mayores recursos y capacidades para la secuenciación genómica ${ }^{3}$.

Los resultados de este análisis parten de una revisión rápida de literatura, cuyas principales limitaciones son la inclusión de solo un motor de búsqueda y la falta de revisión de la calidad de los estudios. Lo anterior, en consideración a la urgencia en la necesidad de información. Seguir la evolución de la epidemia y en particular el comportamiento diferencial de las nuevas variantes resulta fundamental para adaptar las estrategias de respuesta en salud pública, incluyendo las propias medidas de inmunización y su efecto sobre esas VOC.

\section{Conclusión}

Se encuentra más información de la VOC Alpha que de la VOC Gamma. La VOC Alpha reporta mayor riesgo de transmisión, hospitalización y muerte en comparación con otras variantes. Estos resultados deben tomarse con precaución por la naturaleza rápida y narrativa de la presente revisión.

\section{Conflicto de intereses}

Los autores declaran no tener conflictos de intereses.

\section{Referencias}

1. Johns Hopkins University. COVID-19 Map - Johns Hopkins Coronavirus Resource Center [Internet]. https://coronavirus.jhu.edu/map.html

2. Peacock TP, Penrice-Randal R, Hiscox JA, Barclay WS. SARS-CoV-2 one year on: evidence for ongoing viral adaptation. J Gen Virol. 2021 Apr 15;102(4). doi: 10.1099/jgv.0.001584

3. World Health Organization. Weekly epidemiological update on COVID-19 - 4 May 2021 [Internet]. 2021. https://www.who.int/publications/m/item/weeklyepidemiological-update-on-covid-19---4-may-2021

4. World Heealth Organization. COVID-19 new variants: Knowledge gaps and research. 2021; https://www.who.int/publications/m/item/covid-19new-variants-knowledge-gaps-and-research 
5. Instituto Nacional de Salud. Noticias coronavirusgenoma. [cited 2021 May 12]. https://www.ins.gov. co/Noticias/Paginas/coronavirus-genoma.aspx

6. Bager P, Wohlfahrt J, Fonager J, Albertsen M, Yssing Michaelsen T, Holten Møller C, et al. Increased Risk of Hospitalisation Associated with Infection with SARS-CoV-2 Lineage B.1.1.7 in Denmark. SSRN Electron J. 2021 Mar 3 [cited 2021 Apr 28]; doi: 10.2139/ssrn.37928943792894

7. Brookman S, Cook J, Zucherman M, Broughton S, Harman K, Gupta A. Effect of the new SARSCoV-2 variant B.1.1.7 on children and young people. 2020;(January):19-21. doi: 10.1016/S23524642(21)00030-4

8. Docherty A, Harrison E, Semple C. Hospital case fatality and emergence of variant of concern B.1.1.7, rapid CO-CIN report to NERVTAG and SAGE, January 19 th 2021.

9. Ferguson N. Nonparametric analysis of fatal outcomes associated with B1.1.7. 2021; https://assets. publishing.service.gov.uk/government/uploads/ system/uploads/attachment_data/file/961037/ NERVTAG_note_on_B.1.1.7.7_severity_for SAGE_77____pdf

10. Public Health England. Datos no publicados. 2021; https://assets.publishing.service.gov.uk/government/ uploads/system/uploads/attachment_data/ file/961037/NERVTAG_note_on_B.1.1.7_severity_ for_SAGE_77_1_.pdf

11. New and Emerging Respiratory Virus Threats Advisory Group. NERVTAG paper on COVID-19 variant of concern B.1.1.7: paper from the New and Emerging Respiratory Virus Threats Advisory Group (NERVTAG) on new coronavirus (COVID-19) variant B.1.1.7. 2021; https://www. gov.uk/government/\%0Apublications/nervtagpaper-on-covid-19-variant-of-concern-b117

12. Graham MS, Sudre CH, May A, Antonelli M, Murray B, Varsavsky T, et al. Changes in symptomatology, reinfection, and transmissibility associated with the SARS-CoV-2 variant B.1.1.7: an ecological study. Lancet Public Heal. 2021 Apr [cited 2021 Apr 26]; doi: 10.1016/s2468-2667(21)00055-4

13. Brown KA, Gubbay J, Hopkins J, Patel S, Buchan SA, Daneman N, et al. S-Gene Target Failure as a Marker of Variant B.1.1.7 Among SARS-CoV-2 Isolates in the Greater Toronto Area, December 2020 to March 2021. JAMA. 2021 Apr 8 [cited 2021 Apr 28]; doi: 10.1001/jama.2021.5607

14. Volz E, Mishra S, Chand M, Barrett JC, Johnson $\mathrm{R}$, Geidelberg L, et al. Assessing transmissibility of SARS-CoV-2 lineage B.1.1.7 in England. Nature.
2021;(December 2020). doi: 10.1038/s41586-02103470-x

15. Grabowski F, Preibisch G, Giziński S, Kochańczyk M, Lipniacki T. SARS-CoV-2 Variant of Concern 202012/01 Has about Twofold Replicative Advantage and Acquires Concerning Mutations. Viruses. 2021;13(3):1-16. doi: 10.3390/v13030392

16. Grint DJ, Wing K, Williamson E, McDonald HI, Bhaskaran K, Evans D, et al. Case fatality risk of the SARS-CoV-2 variant of concern B.1.1.7 in England, 16 November to 5 February. Euro Surveill. 2021;26(11). doi: 10.2807/1560-7917. ES.2021.26.11.2100256

17. Frampton D, Rampling T, Cross A, Bailey H, Heaney J, Byott M, et al. Genomic characteristics and clinical effect of the emergent SARS-CoV-2 B.1.1.7 lineage in London, UK: a whole-genome sequencing and hospital-based cohort study. Lancet Infect Dis. 2021 Apr [cited 2021 Apr 28]; doi: 10.1016/S1473-3099(21)00170-5

18. Davies NG, Jarvis CI, Edmunds WJ, Jewell NP, Diaz-Ordaz K, Keogh RH, et al. Increased mortality in community-tested cases of SARSCoV-2 lineage B.1.1.7. 2021 [cited 2021 Apr 28];2021.02.01.21250959. doi: 10.1038/s41586021-03426-1

19. Davies NG, Davies NG, Abbott S, Barnard RC, Jarvis CI, Kucharski AJ, et al. Estimated transmissibility and impact of SARS-CoV-2 lineage B.1.1.7 in England. Science (80- ). 2021;3055(March):1-16. doi: $10.1126 /$ science.abg3055

20. Calistri P, Amato L, Puglia I, Cito F, Di Giuseppe A, Danzetta ML, et al. Infection sustained by lineage B.1.1.7 of SARS-CoV-2 is characterised by longer persistence and higher viral RNA loads in nasopharyngeal swabs. Int $\mathrm{J}$ Infect Dis. Apr [cited 2021 Apr 28];105: 753-755. doi: 10.1016/j. ijid.2021.03.005

21. Challen R, Brooks-Pollock E, Read JM, Dyson L, Tsaneva-Atanasova K, Danon L. Risk of mortality in patients infected with SARS-CoV-2 variant of concern 202012/1: Matched cohort study. BMJ. 2021; 372: 1-10. doi: 10.1136/bmj.n579

22. Loconsole D, Centrone F, Morcavallo C, Campanella S, Sallustio A, Accogli M, et al. Rapid Spread of the SARS-CoV-2 Variant of Concern 202012/01 in Southern Italy (December 2020-March 2021). Int J Environ Res Public Health. 2021 Apr 29 [cited 2021 May 11];18(9). doi: 10.3390/ijerph18094766

23. Garvey MI, McMurray C, Casey AL, Ratcliffe L, Stockton J, Wilkinson MAC, et al. Observations of SARS-CoV-2 variant of concern B.1.1.7 at the UK's 
largest hospital Trust. J Infect. 2021 Apr 28 [cited 2021 May 11]; doi: 10.1016/j.jinf.2021.04.026

24. Zimerman RA, Cadegiani FA, Pereira e Costa RA, Goren A, Campello de Souza B. Stay-AtHome Orders are associated with emergence of novel SARS-CoV-2 Variants. Cureus. $2021 \mathrm{Mar}$ 11 [cited 2021 Apr 28];13(3): 1-12. doi: 10.7759/ cureus. 13819

25. Datos no publicados. 2021; https://assets.publishing. service.gov.uk/government/uploads/system/ uploads/attachment_data/file/961037/NERVTAG_ note_on_B.1.1.7_severity_for_SAGE_77_1_.pdf

26. New and Emerging Respiratory Virus Threats Advisory Group. NERVTAG paper on COVID-19 variant of concern B.1.1.7: paper from the New and Emerging Respiratory Virus Threats Advisory Group (NERVTAG) on new coronavirus (COVID-19) variant B.1.1.7. 2021. 